\title{
A CASE OF CHILDHOOD AUTOIMMUNE POLYGLANDULAR SYNDROME TYPE I
}

\section{Dr.Aysegul Alpcan ${ }^{1}$, Dr.Bilge Eris² ${ }^{2}$ Dr.Senay Arikan Durmaz ${ }^{3}$, Dr.Aydin Cifci², Dr.lsilay Kalan ${ }^{3}$}

${ }^{1}$ Kirikkale Yuksek Ihtisas Hospital, Kirikkale,Turkey

2Department of Internal Medicine, Kirikkale University, Kirikkale, Turkey

${ }^{3}$ Department of Endocrinology and Metabolism, Kirikkale University, Kirikkale, Turkey

\section{INTRODUCTION}

Autoimmune polyglandular syndromes (APS) are rare groups of diseases with autoimmune failure of at least two endocrine glands. Only endocrine glands may be affected as well as autoimmune diseases involving other organs may accompany. In general, APSI first occurs with candidiasis. Hypoparathyroidism generally presents until 10 years of age, and adrenal insufficiency insert around 20 years. Usually all findings are completed at 40 years old. Here, a young girl is the first presented early onset APS type I with adrenal insufficiency.

\section{CASE REPORT}

White plaques on the tongue and oral mucosa suggestive of mucocutaneous candidiasis and yellow and thick ectodermal dystrophic changes (Figure 1 and 2) particularly in the hand nails attracted attention in an 18-year-old female patient in asthenic appearance who attended the Endocrinology outpatient clinic of Kirikkale University Medical School for malaise and failure to thrive. No hyperpigmantation was detected on the skin and mucosa on physical examination. Pubertal development was accordant with Tanner Stage 5. The skin was dry. Other systemic examinations were normal.

It was learned from her personal history that malaise, loss of appetite, darkening in skin color, and changes in the nails were first detected and investigated at a Pediatrics outpatient clinic she attended when she was six years old. As much as learned from her records at that time, her height and weight were consistent with her age, turgor was decreased, the skin was dry, there were marking, shape and color changes in the hand nails. Candidiasis and skin hyperpigmentation were detected.

As PTH was 2,83 pg/ml (15-65), calcium 8,5mg/dl, ALP 183 $\mathrm{U} / \mathrm{I}, \mathrm{P} 69 \mathrm{mg} / \mathrm{dl}(4,5-5,5)$, ACTH was $1250 \mathrm{pg} / \mathrm{ml}$ (7.2-63.3), and basal cortisol was $1 \mu \mathrm{g} / \mathrm{dl}(6.2-19.4)$ in the blood tests, and there was no response to Synacthen Stimulation Test. Primary adrenal insufficiency, idiopathic hypoparathyroidism and candidiasis were detected and the patient was diagnosed for APS type I. Celiac antibodies were found to be negative.

The treatment of the patient was stareted at the pediatric clinic, but she was referred to our clinic as having entered adulthood. When the patient consulted our clinic, she was taking $10 \mathrm{mg}$ hydrocortisone 4 times a day, $0.1 \mathrm{mg}$ fludrocortisone a day, $1000 \mathrm{mg}$ calcium carbonate a day and $0,5 \mathrm{mg}$ calcitirol twice a day

\section{CONCLUSION}

APS usually occurs in childhood and autosomal recessive disorder characterized by a combination of autoimmune manifestations affecting endocrine and non-endocrine organs. APS is rare disease but important to recognize and treat early to avoid significant morbidity and mortality.

APSs clinically include a quite broad group of diseases. When one type of endocrinologic disease is detected in a patient, the patient should be tested for complete blood count, electrolytes, hormonal levels, and antibodies for other diseases once every three years.

\section{Figure 1}

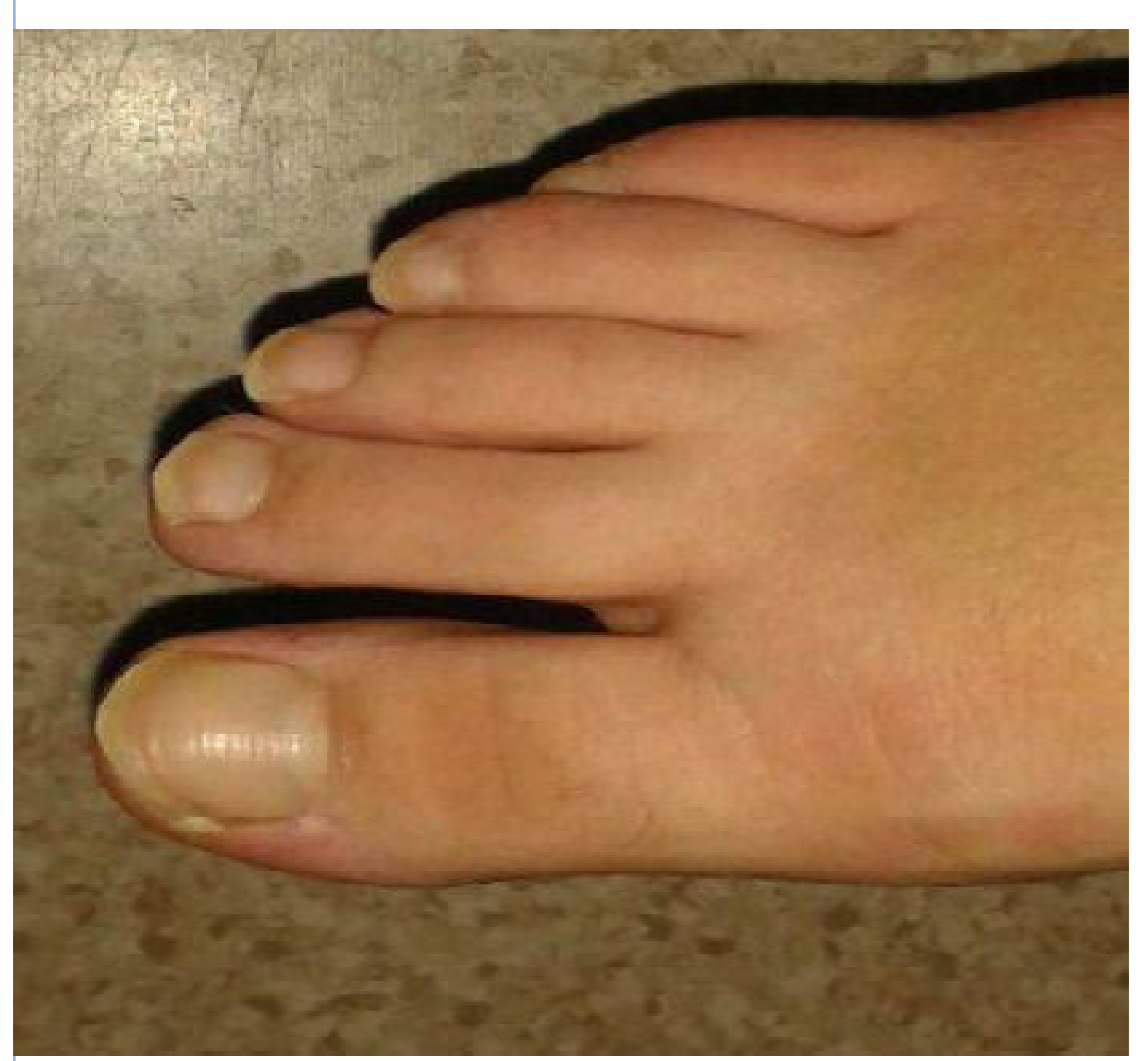

Figure 2

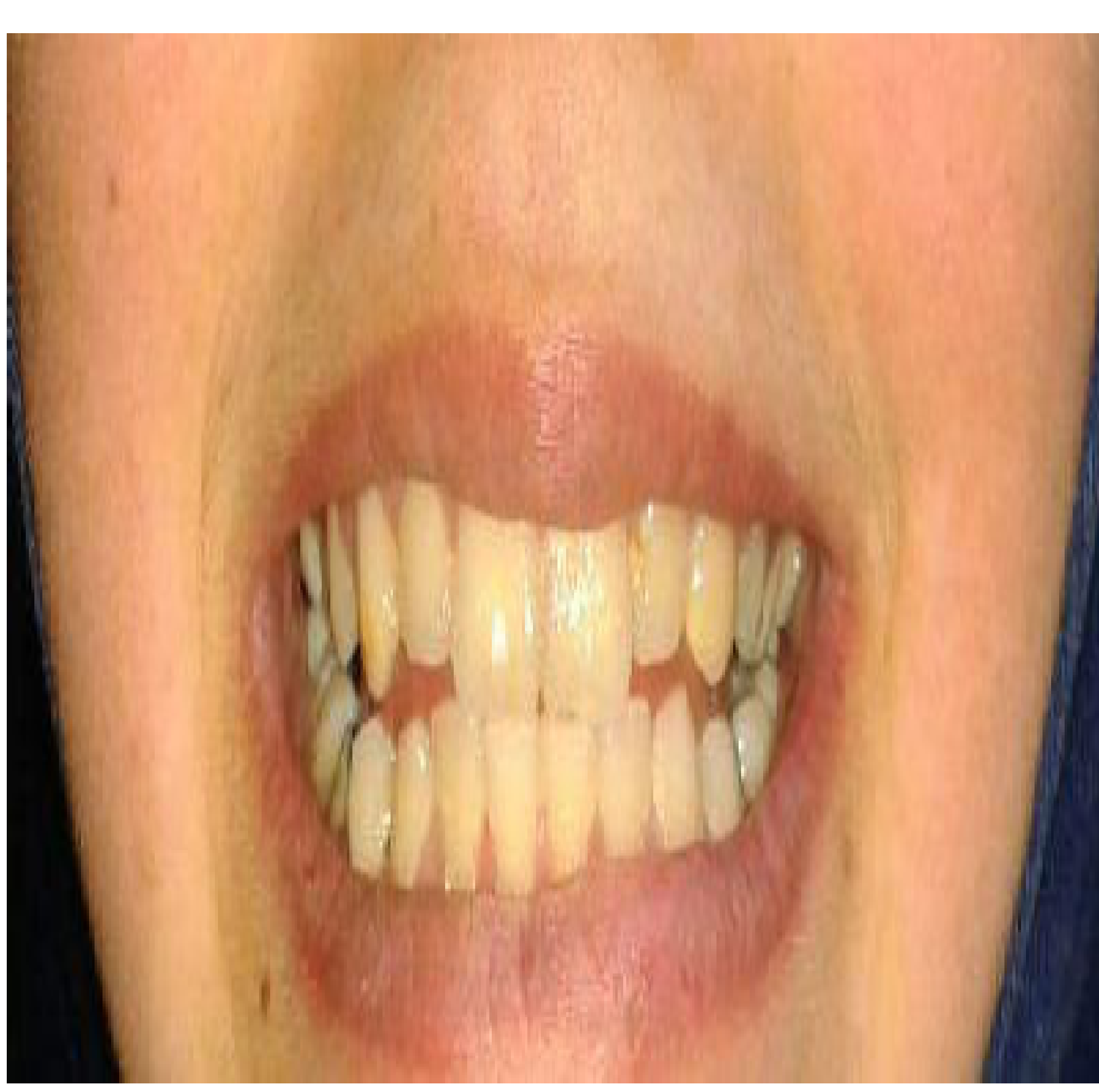

